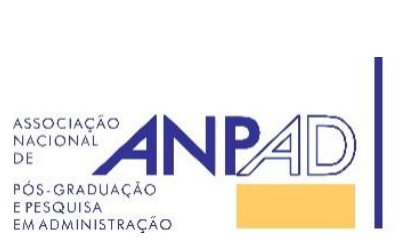

Disponível em

http://www.anpad.org.br/rac

RAC, Rio de Janeiro, v. 22, n. 3, art. 5, pp. 403-423, maio/junho, 2018, http://dx.doi.org/10.1590/1982-7849rac2018170223

$(\mathrm{cc}) \mathrm{EY}$

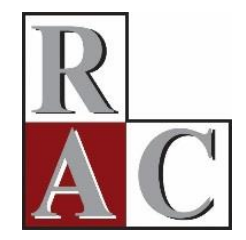

\title{
O Uso de Biomarcadores em Pesquisas de Marketing
}

\author{
The Use of Biomarkers in Marketing Research
}

Cristina Maria de Aguiar Pastore ${ }^{1}$ Eliane Cristine Francisco Maffezzolli ${ }^{1}$ José Afonso Mazzon²

Pontifícia Universidade Católica do Paraná, Escola de Negócios, Curitiba, PR, Brasil ${ }^{1}$ Universidade de São Paulo, Faculdade de Economia, Administração e Contabilidade, São Paulo, SP, Brasil ${ }^{2}$ 


\title{
Resumo
}

A aproximação do marketing com a neurociência tem gerado expectativa tanto na academia quanto no mercado, em especial na última década. Apesar do interesse e das promessas de resultados, lacunas de conhecimento neurobiológico são percebidas acerca de formas de coleta e análise de dados. Este estudo apresenta e compara algumas ferramentas neurocientíficas que são fundamentadas em respostas não cognitivas do cérebro humano. A coleta e a análise de biomarcadores, como hormônios e níveis de atividade elétrica muscular, podem ser utilizadas em pesquisas de comportamento humano relacionadas ao consumo ou em outras interações com ações de marketing. A metodologia de aplicação dessas ferramentas, medidas resultantes e possíveis interpretações de comportamento também são discutidas, com ênfase em futura agenda de pesquisa.

Palavras-chave: biomarcadores; comportamento do consumidor; neurociência.

\begin{abstract}
The crossovers between marketing and neuroscience has generated expectations as much for academia as for the market, especially in the last decade. Despite the interest and promises of results, gaps in neurobiological knowledge are perceived related to data collection and analysis. This study presents and compares some neuroscientific tools that are based on non-cognitive responses of the human brain. The collection and analysis of biomarkers such as hormones and levels of muscular electrical activity can be used in research on human behavior related to consumption, among other interactions with marketing actions. We also discuss the methodological application of these tools, resulting measures and possible interpretations of behavior, with emphasis on future research agenda.
\end{abstract}

Key words: biomarkers; consumer behavior; neuroscience. 


\section{Introdução}

O uso de técnicas de autorrelato para pesquisas de comportamento humano possui, como toda forma de coleta de dados, vantagens e desvantagens. Dentre as últimas, estão dois problemas decorrentes do processamento cognitivo humano: não quero responder sobre isto, por diversas razões, que incluem filtros éticos e morais; ou não posso, porque não tenho consciência das minhas reais motivações sobre este assunto. A racionalidade humana, importante para adaptação do sujeito ao meio e sua sobrevivência neste, pode comprometer a verbalização de motivações reais ou impedir pesquisadores de conhecê-las. A coleta de dados com biomarcadores em pesquisas de comportamento humano busca transpor essa dificuldade, colhendo informações independentes da cognição e da racionalidade humanas (Kenning \& Plassmann, 2008).

Os conceitos e as aplicações da neurociência na psicologia do consumo ganharam popularidade na última década, tanto na pesquisa acadêmica quanto na gestão de marketing. O número de artigos publicados em top journals, a busca de referências no Google acadêmico sobre esse tópico, e a quantidade de empresas oferecendo serviços relacionados cresceram exponencialmente nesse período (Plassmann, Ramsøy, \& Milosavljevic, 2012). Ainda que o interesse tenha aumentado, permanecem dúvidas sobre quando e de que maneira conduzir a coleta e a análise de dados biológicos para investigar comportamentos. Tal situação pode ser compreendida, em parte, pela pouca maturidade de pesquisas na área e pela insuficiência de equipes multidisciplinares que contemplem diferentes áreas de conhecimento.

Um dos motivos pelos quais a neurociência aparentou ser uma fonte relevante para pesquisadores de administração e, em especial, de marketing, é a possibilidade que oferece de investigar respostas fisiológicas. Sendo resultados de processos neurais subconscientes ou inconscientes, a análise dessas variáveis elimina o filtro do processamento cognitivo do sujeito sobre suas respostas a questionamentos. Existe, entretanto, uma importante observação a ser feita: como tudo o que é novo e advindo de outro campo de estudo, podem ocorrer erros de compreensão e conclusões equivocadas sobre esses dados. Há nesses, como em quaisquer outros métodos investigativos, vantagens e desvantagens; há situações nas quais são aplicáveis e outras em que não são, e, principalmente, como em qualquer outra circunstância de pesquisa, os modelos neurocientíficos escolhidos precisam ser adequados às variáveis e ao contexto de pesquisa investigados.

Na tentativa de amadurecer a aproximação da neurociência com as ciências sociais aplicadas, em busca de um consenso e de uma construção teórica, alguns autores propuseram nomes distintos para as investigações. Dentre elas, a Neurociência Cognitiva Organizacional se propôs a ser uma maneira de pensar sobre o negócio que modela a interação entre os sistemas biológicos básicos do cérebro, como as emoções, os sistemas de processamento de informações cognitivas em que se encontra a racionalidade humana, e os processos que consideram interações sociais, objetivando entender o negócio como um processo fundamentalmente biológico (Lee, Butler, \& Senior, 2010). A chamada Neurociência da Decisão procura compreender os mecanismos neurais pelos quais acontecem as decisões humanas, sejam econômicas, de consumo ou de outras origens (Shiv et al., 2005). A Neuroeconomia, por sua vez, propõe ser a área que estuda as decisões financeiras sob o prisma neurobiológico, e que contempla o Neuromarketing, ou o estudo das decisões de consumo (Hubert, 2010).

No que diz respeito especificamente ao marketing, passada a expectativa desvirtuada de finalmente compreender a mente do consumidor, é proposto que o título Neurociência do Consumidor passe a ser aquele utilizado para descrever o uso de conhecimentos e ferramentas neurobiológicas neste contexto. Para alguns autores (Javor, Koller, Lee, Chamberlain, \& Ransmayr, 2013; Plassmann et al., 2012; Yoon et al., 2012), o objetivo da Neurociência do Consumidor é adaptar criteriosamente métodos e teorias da neurociência, combinados com teorias comportamentais, modelos e designs experimentais testados originados na psicologia do consumidor, além de áreas como ciências da decisão, para desenvolver uma abordagem que ajude os pesquisadores a entenderem elementos mais profundos do comportamento do consumidor. Por ser um termo abrangente, que considera o corpo 
teórico já construído dentro da psicologia do consumo, o título Neurociência do Consumidor parece plausível. Nas palavras de Javor, Koller, Lee, Chamberlain e Ransmayr (2013, p. 8): "Nós argumentamos em favor de uma terminologia diferente, chamando aplicações comerciais de métodos neurocientíficos de "neuromarketing', e as aplicações científicas, de "neurociência do consumidor"'.

Entretanto, além da discussão semântica, o foco está em compreender o que é a neurociência e como ela contribui para o corpo teórico do marketing. Devem ser definidos quais métodos advindos da área são interessantes ao estudo teórico-empírico do marketing, quando utilizá-los e com qual finalidade. Na delimitação do escopo, a neurociência é o estudo do sistema nervoso que procura entender aspectos biológicos fundamentais do comportamento. Seu objeto de estudo envolve desde células individuais e das moléculas que as compõem (neurociência celular) até sistemas cerebrais complexos e a forma pela qual eles interagem entre si, gerando comportamentos e respostas fisiológicas que vão além do sistema nervoso central (Lent, 2004). Tratar da Neurociência do Consumidor envolve saber em que pontos dessa vasta dimensão neurocientífica os conhecimentos são relevantes à compreensão do comportamento do consumidor.

O objetivo principal deste estudo é mapear e comparar diferentes marcadores biológicos de respostas autonômicas - respostas não cognitivas, secundárias a estímulos neuronais - enquanto medidas de comportamento em pesquisas de marketing, apresentando ferramentas de pesquisa neurocientíficas e suas aplicações. A literatura existente tem se concentrado em conceitos e aplicações de ferramentas de neuroimagem, como a ressonância magnética funcional (fMRI), a atividade elétrica neural como o eletroencefalograma (EEG) e/ou o mapeamento do movimento ocular (eye-tracker), aplicados ao comportamento do consumidor. Porém, pouco ainda se fala sobre o uso de medidas de níveis hormonais, de neurotransmissores e outras substâncias circulantes no organismo humano, de respostas secundárias a essas, tais quais pressão arterial, frequência cardíaca e respiratória, condutância elétrica da pele ou a intensidade de disparos elétricos musculares, ainda que sejam sabidamente reflexos de comandos neuronais (Lent, 2004), especialmente se considerados a facilidade e o baixo custo de coleta comparados a outros métodos.

Por trazer métodos e possibilidades novas de pesquisa ao campo de marketing, a aproximação com a neurociência contribui muito com a investigação, em especial sobre o comportamento do consumidor, permitindo novas abordagens a pesquisas que classicamente são investigadas a partir de relatos e observações. Fatores de decisão, eficácia publicitária, interação entre estímulos internos e externos e diversos outros objetos de investigação no marketing ganham força teórica quando são consideradas também as possibilidades advindas da neurociência. As sessões subsequentes deste artigo apresentam elementos conceituais sobre a ativação autonômica e arousal, o comparativo dos métodos de mensuração de dados neurobiológicos, principais aplicações e agenda de pesquisa.

\section{A Origem dos Dados Neurobiológicos e a Comparação dos Métodos de Mensuração}

O ponto de partida para diversas medidas autonômicas é o que pode ser traduzido por nível de excitação do organismo humano, ou em inglês, arousal. Níveis diferentes de excitação estão intimamente ligados à atenção e tornam o organismo mais sensível a estímulos relevantes, enquanto os irrelevantes são filtrados e não processados. A região do cérebro que origina tal resposta, nomeada Formação Reticular (RF), comanda várias respostas de excitação que estão relacionadas, como frequência cardíaca e frequência respiratória, pressão arterial e condutância elétrica da pele. Essas respostas podem incluir ainda a ativação de outras áreas responsáveis por processamentos como emoções (amígdala) ou memória (hipotálamo) (Groeppel-Klein, 2005). É importante destacar que a excitação autonômica não possui valência afetiva, estando presente em situações agradáveis e desagradáveis. Também podem ser classificadas como de alta excitação, como o estado de surpresa, ou de baixa excitação, como os estados de sonolência e cansaço (Weierich, Wright, Negreira, Dickerson, $\&$ Barrett, 2010). 
As principais formas de coleta de dados originadas da neurociência se dividem em três grandes grupos: (a) coletam informações sobre a atividade metabólica do cérebro; (b) sobre a atividade elétrica do cérebro; ou (c) coletam informações secundárias, decorrentes da atividade neural, como frequência cardíaca ou diâmetro das pupilas (Butler, 2008; Habel et al., 2007; Javor et al., 2013; Kenning \& Plassmann, 2005; Zurawicki, 2010). Os registros de atividade metabólica e elétrica no cérebro são as medidas mais encontradas em estudos de neurociência na administração, enquanto há pouca discussão sobre o uso de respostas secundárias a comandos neuronais, os quais possuem formas de mensuração mais simples e práticas. A Figura 1 ilustra algumas dessas formas de coleta, com suas metodologias próprias e medidas decorrentes. Também ilustra um quarto grupo de medidas que não são decorrentes de estímulos neuronais, mas que podem ser bastante úteis nesse tipo de investigação. Aplicações de pesquisa para essas medidas estão expostas na seção Principais Aplicações em Marketing.

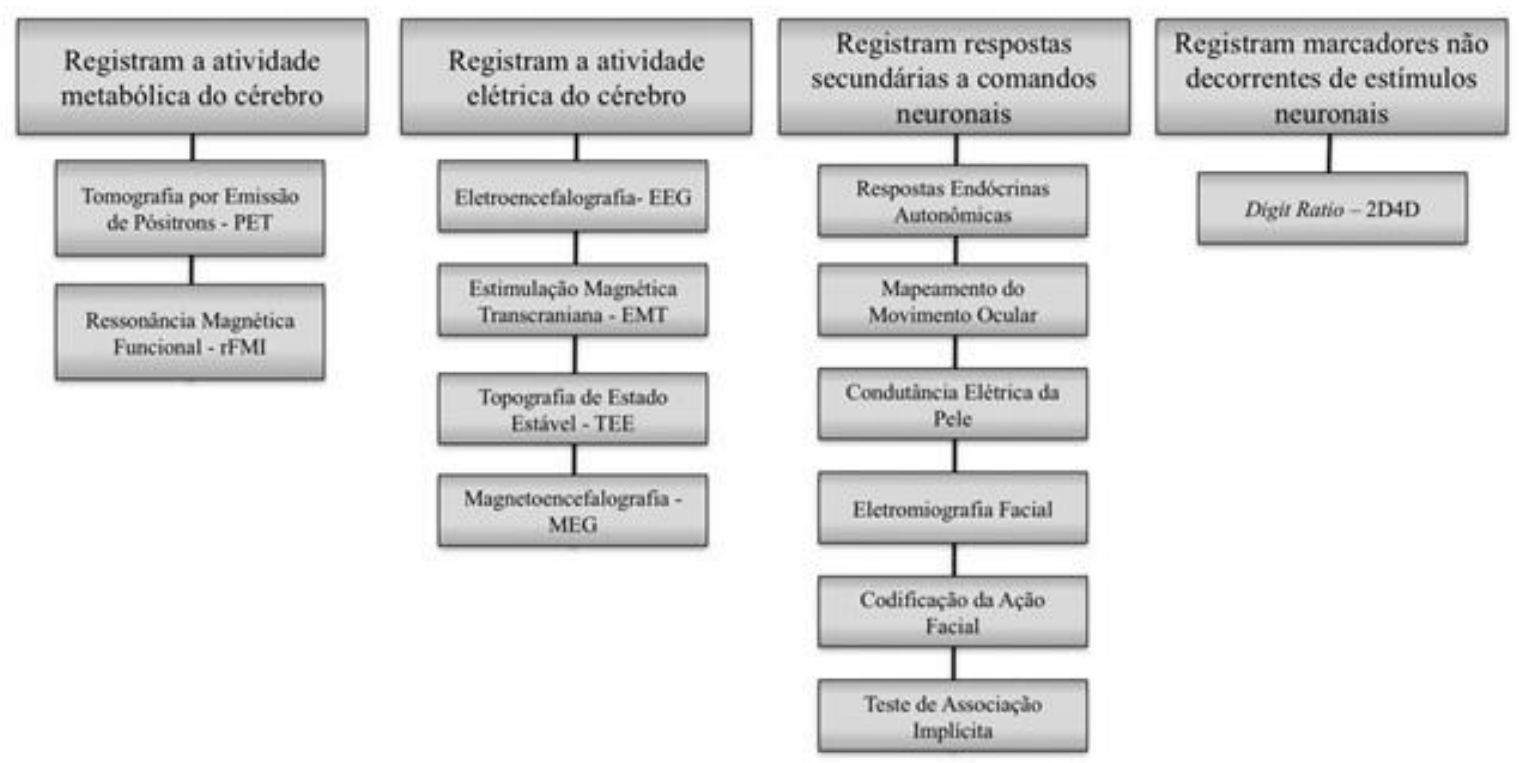

Figura 1. Principais Ferramentas de Coleta de Dados Neurobiológicos e/ou Biológicos Fonte: Elaborada pelos autores de acordo com Lent, R. (2004). Cem bilhões de neurônios? Conceitos fundamentais de neurociência (2a ed., p. 499). Rio de Janeiro: Atheneu.

As formas de coleta de dados neurobiológicos se diferenciam por conta do objeto medido e também em características operacionais como tempo - de coleta e para acessar os resultados -, custos financeiros, interpretação, incidência de fatores influenciadores, outliers, dentre outros. As medidas secundárias são em essência mais simples de serem coletadas e interpretadas e, dessa forma, estão mais difusas em centros de diagnóstico pelo país e de acesso mais fácil a pesquisadores de marketing. Exames de atividade metabólica ou elétrica do cérebro, como Tomografia, Ressonância Magnética Funcional ou Eletroencefalografia, possuem operacionalização mais complexa e, portanto, são mais caros e menos acessíveis aos pesquisadores de marketing em geral. A Tabela 1 apresenta um comparativo entre as formas de coleta de acordo com sua operacionalização. 
Tabela 1

Diferenças Operacionais na Forma de Coleta de Dados Neurobiológicos

\begin{tabular}{lcccc}
\hline EXAME & TEMPO & TEMPO & CUSTO & ESPECIALISTAS* \\
\hline PET & +++ & + & +++ & +++ \\
\hline rFMI & +++ & + & +++ & +++ \\
\hline EEG & +++ & + & +++ & +++ \\
\hline EMG & +++ & + & +++ & +++ \\
\hline TEE & +++ & + & +++ & +++ \\
\hline MEG & +++ & + & ++ & +++ \\
\hline Respostas Endócrinas & + & ++ & + & ++ \\
\hline Movimento Ocular & ++ & + & + & ++ \\
\hline Cond. Elétrica da Pele & ++ & + & + & ++ \\
\hline Eletromiografia Facial & ++ & + & + & ++ \\
\hline Ação Facial & ++ & ++ & + & ++ \\
\hline Associação Implícita & + & + & + & ++ \\
\hline 2D4D & + & + & + & + \\
\hline
\end{tabular}

Nota. Fonte: Elaborada pelos autores.

*Necessidade de profissionais especializados para a leitura/interpretação dos resultados, que podem ser das áreas médica, de engenharia, estatística ou outra. **As informações de operacionalização dos exames foram encontradas em: AbcMed - Informações sobre sua Saúde. (2015, janeiro 21). PET Scan: o que é? Por que fazer? Como é feita? Quem deve e quem não deve fazer? Quais são as possiveis complicações? Recuperado em 7 julho, 2016, de http://www.abc.med.br/p/exames-eprocedimentos/740267/pet+scan+o+que+e+por+que+fazer+como+e+feita+quem+deve+e+quem+nao+deve+fazer+quais+sao+as+p ossiveis+complicacoes.htm; AbcMed - Informações sobre sua Saúde. (2013 setembro 20). Eletroencefalograma: como é feito? Como se preparar para o exame? Quais são as complicações? Recuperado em 7 julho, 2016, de http://www.abc.med.br/p/exames-eprocedimentos/347519/eletroencefalograma+como+e+feito+como+se+preparar+para+o+exame+quais+sao+as+complicacoes.htm; AbcMed - Informações sobre sua Saúde. (2013 setembro 30). Nevralgia ou neuralgia: o que é isso? Recuperado em 7 julho, 2016, de http://www.abc.med.br/p/sinais.-sintomas-e-doencas/369059/nevralgia+ou+neuralgia+o+que+e+isso.htm; e Centro Médico Sorocaba. (n.d.). Exames. Recuperado em 7 julho, 2016, de https://www.centromedicosorocaba.com.br/exames

\section{Condutividade elétrica da pele}

Uma das maneiras de medir a resposta autonômica de excitação no organismo humano é, por meio da sua atividade eletrodermal, termo geral usado para definir mudanças nas propriedades elétricas da pele. A atividade eletrodermal mais conhecida e estudada é a condutância elétrica, quantificada pela medida do fluxo elétrico resultante da aplicação de um potencial elétrico entre dois pontos de contato com a pele (Braithwaite, Watson, Jones, \& Rowe, 2013).

Durante respostas autonômicas de atividade simpática, tais como emocionais, ocorre a liberação na superfície da pele de uma sudorese fina chamada sudorese écrina, manifestada na palma das mãos e na sola dos pés (Lent, 2004). Por conta da composição do suor, a condutividade elétrica da pele com sudorese é maior que aquela sem o suor, tornando, então, a condutividade uma medida fisiológica da intensidade da resposta de excitação autonômica (arousal) do indivíduo (Zurawicki, 2010), e também a mais sensível das avaliações possíveis, capaz de registrar pequenas variações (Groeppel-Klein, 2005). Com objetivo de reafirmar a superioridade de medidas biológicas às medidas de percepção para a avaliação de arousal, a autora ainda destaca que

a validade da medida verbal de arousal é questionada: métodos de autorrelato somente permitem medidas posteriores no tempo. Além disso, a seleção inapropriada de itens para representar o 
arousal percebido é criticado, e as respostas verbais requerem esforço cognitivo do indivíduo, que pode não ter consciência do arousal (Groeppel-Klein, 2005, p.1).

A coleta de dados da condutância elétrica da pele acontece de maneira simples: dois eletrodos são acoplados, com um pouco de gel condutor, em diferentes pontos da pele das mãos, medindo-se, então, o fluxo elétrico entre dois pontos. Quanto maior a sudorese, maior o fluxo da corrente entre os pontos (Koller \& Walla, 2012; Zurawicki, 2010).

\section{Frequência cardíaca, respiratória e pressão arterial}

Outras medidas de resposta autonômica incluem frequência cardíaca, frequência respiratória e pressão arterial. O sistema nervoso autônomo age sobre o sistema cardiovascular, regulando, a todo momento, a pressão arterial, através de batimentos cardíacos, calibre e tensão dos vasos sanguíneos. Respostas de ativação do sistema autonômico simpático geram aumento da pressão arterial e da frequência cardíaca, assim como o oposto na ativação parassimpática (Lent, 2004). A pressão arterial sanguínea é a medida da pressão que o sangue exerce ao ser bombeado pelo coração, e pode sofrer influência do volume de líquido circulante dentro do vaso, do calibre do vaso, ou da força com que os músculos do coração se contraem, impulsionando o sangue para circular pelo corpo.

A medida indireta da pressão arterial se dá pela compressão de uma artéria importante do braço (braquial) na altura do pulso ou do cotovelo, com um aparelho chamado esfignomanômetro, por meio de técnicas auscultatórias (algo semelhante a ouvir a pulsação da artéria), ou oscilométricas (observando a oscilação de uma agulha ou ponteiro, produzida pela pressão do sangue na artéria), e que podem ser manuais ou automatizadas (Perloff, Grim, Flack, \& Frohlich, 1993).

Os equipamentos para aferição de pressão arterial (PA), frequência cardíaca (FC) e respiratória (FR) são bastante simples e variados. Há modelos em formato de bracelete para PA e FC, outros como fitas com sensores para serem acoplados ao peito e relógios com leitores para as diversas medidas, marcadores acoplados aos dedos para FC e diversos outros, de transmissão wireless ou não. A coleta é simples, não invasiva e contínua, o que permite ao pesquisador observar variações decorrentes de respostas autonômicas durante a exposição de estímulos, em tempo real. As frequências de coleta, em hertz $(\mathrm{Hz})$, também variam conforme o fabricante e o equipamento; portanto, o ponto chave para o uso adequado dessa ferramenta é a sincronização de dados coletados em diferentes frequências.

A influência de fatores ambientais sobre os resultados também deve ser cuidadosamente controlada. Barulhos abruptos, por exemplo, podem gerar aumentos bruscos e repentinos de algumas medidas por estímulos autonômicos, o mesmo acontecendo com o nível e a variação de temperatura, luminosidade e diversos outros. A análise estatística desses dados requer rigor metodológico para a distinção de medidas que são outliers daquelas decorrentes do estímulo analisado, identificando amplitudes basais, esperadas e discrepantes nos indicadores.

\section{Potencial elétrico muscular}

A eletromiografia de superfície possibilita medir respostas secundárias a comandos neuronais não cognitivos baseadas na atividade de contração de determinados grupos musculares. O registro dos padrões desses potenciais de ação é denominado eletromiografia - EMG - e traduz a atividade elétrica muscular (Criswell, 2011). Esse procedimento não é invasivo, os eletrodos são acoplados à pele com um gel condutor e, portanto, os riscos ao paciente são mínimos.

A utilização da EMG em pesquisas de comportamento de consumo está, em parte, direcionada para a identificação fisiológica de expressões faciais relacionadas à expressão de emoções básicas como felicidade, raiva, medo, desgosto, dentre outras, assim como de níveis de arousal (Boxtel, 2010). A base para a relação entre contração muscular facial e emoções está nos estudos de Paul Ekman, que se dedicou à análise de expressões faciais e emoções subjacentes. Segundo o autor, alguns músculos da face - como o orbicular do olho e o zigomático maior - estão relacionados à felicidade, em que contrações elevam 
os cantos da boca e reduzem a abertura dos olhos, fazendo a pessoa sorrir. Outros - frontal e levantador da pálpebra superior - estão ativos em casos de surpresa, uma medida de excitação que não possui valência afetiva, aumentando a abertura do olho e erguendo as sobrancelhas (Ekman \& Friesen, 1982; Ekman \& Rosenberg, 1997; Ekman, 2006). Cabe aqui destacar que emoção é um constructo cuja essência não possui consenso acadêmico (Mesquita, 2016). O ponto comum entre autores é o envolvimento de diversos sistemas do organismo humano que não podem ser analisados em sua plenitude por meio de apenas um ou outro indicador (Kreibig, 2010).

O mapeamento das expressões faciais relacionadas às emoções também é a base para a comparação realizada por softwares sobre a expressão realizada pelo sujeito em análise e o estado básico padrão. Hill (2003) foi um dos pioneiros na sua aplicação em pesquisas de marketing, procurando entender o papel das emoções no processo de consumo. Equipamentos de leitura facial (face reader) permitem coleta de dados não invasiva, sem a necessidade de eletrodos ou outros leitores, além de uma câmera de vídeo capaz de captar as expressões faciais do participante da pesquisa e, por isso, mostra-se uma ferramenta bastante acessível para o mapeamento de emoções básicas. A confiabilidade dos dados depende da calibração do equipamento, da amplitude das expressões faciais do sujeito - bastante distinta em diferentes etnias - e de possíveis fontes de interferência (Yan, Andrews, Jenkins, \& Young, 2016).

Além de expressões faciais relacionadas a emoções, o reflexo de sobressalto de susto, ou startle reflex, também pode ser um indicador válido e de coleta simples para pesquisas de comportamento, baseado em dados eletromiográficos. Enquanto a medida de condutância elétrica da pele é conhecida por sua sensibilidade à excitação, o reflexo de sobressalto é conhecido por sua sensibilidade à valência afetiva. Aparentemente, o disparo elétrico muscular para causar abertura ocular (uma resposta autonômica) é mais intenso em casos de estímulos afetivos negativos que em positivos, e é medido a partir de três eletrodos posicionados na face do participante, na altura de músculos específicos, que captam a atividade elétrica muscular. A condutância elétrica da pele e o reflexo do sobressalto podem ser combinados para registar simultaneamente aspectos de valência e excitação decorrentes de respostas autonômicas (Koller \& Walla, 2012).

\section{Hormônios}

Especificamente, a noradrenalina (um neurotransmissor) e a adrenalina (o hormônio liberado a partir do primeiro) são biomarcadores para a ativação do sistema autonômico bastante promissores em pesquisas de comportamento. Além de suas respostas secundárias já citadas, os níveis circulantes das substâncias podem ser aferidos diretamente. O nível de noradrenalina pode ser obtido a partir do plasma sanguíneo, enquanto o hormônio adrenalina pode ser medido através de pequenas quantidades coletadas de urina dos participantes, por exemplo, em experimentos de laboratório (Dart, Du, \& Kingwell, 2002). A possibilidade de medir níveis hormonais além da expressão sanguínea é uma grande facilidade para pesquisas de comportamento, reduzindo drasticamente a característica invasiva da coleta. Há estudos relatando a medida feita a partir de fios de cabelo e amostras de saliva ou urina (Javor et al., 2013).

\section{Digit Ratio ou 2D:4D}

Uma medida biológica não decorrente de estimulação neural aguda, mas que está ganhando espaço nas publicações de pesquisa em administração e, portanto, merece destaque, é a medida chamada 2D:4D, ou digit ratio (Nepomuceno, Saad, Stenstrom, Mendenhall, \& Iglesias, 2016). Trata-se da razão entre o tamanho do segundo dedo da mão direita - popularmente chamado de indicador - e o quarto dedo da mesma mão - ou dedo anelar. O 2D:4D é uma medida reconhecida na literatura para a exposição pré-natal aos hormônios testosterona e estradiol (Austin, Manning, McInroy, \& Mathews, 2002; Lutchmaya, Baron-Cohen, Raggatt, Knickmeyer, \& Manning, 2004). Em termos objetivos, essa exposição do feto aos hormônios circulantes na placenta materna, durante a gestação, influencia na formação de todo o seu organismo, principalmente o cérebro e, por consequência, em traços de comportamento futuro (Fink, Manning, \& Neave, 2004). 
A medida é simples e feita a partir de um paquímetro digital de alta precisão. Com a divisão dos valores, forma-se um índice, diretamente relacionado à exposição de estradiol e inversamente proporcional à exposição de testosterona: mulheres tendem a apresentar índices maiores que homens, todos variando em torno de 1. Embora seja uma medida de característica crônica e que não oscila conforme estímulos específicos, o 2D:4D tem se mostrado promissor na busca pela compreensão de determinados comportamentos de decisão, sejam financeiros ou de consumo (Coates, Gurnell, \& Rustichini, 2009; Fink, Neave, Laughton, \& Manning, 2006; Pastore, Nepomuceno, Stenstrom, \& Francisco-Maffezzolli, 2016; Stenstrom, Saad, Nepomuceno, \& Mendenhall, 2011).

\section{Principais Aplicações em Marketing}

Entendidas as bases fisiológicas e da coleta de dados, esta seção se destina a expor algumas aplicações práticas já realizadas utilizando os métodos discutidos. Os estudos servem de bases e de diretrizes para pesquisas futuras.

Com o objetivo de diferenciar os efeitos causados por fama e beleza no contexto publicitário sobre a resposta autonômica, medidas de condutância elétrica da pele de 24 mulheres jovens destras foram coletadas enquanto elas avaliavam anúncios de perfumes endossados por modelos que eram famosos/não famosos ou atraentes/não atraentes. Os dados fisiológicos foram aferidos enquanto as mulheres observavam os anúncios, e os resultados sugerem que fama gera respostas de arousal mais intensas que beleza. Celebridades não atraentes evocaram respostas de excitação significativamente maiores que desconhecidos bonitos, no contexto estudado. Além disso, as respostas foram mais agudas na mão esquerda das participantes, sugerindo uma ativação maior do hemisfério direito do cérebro, local onde prioritariamente as emoções são processadas (Gakhal \& Senior, 2008).

Além da condutância elétrica da pele, dados de eletromiografia facial e frequência cardíaca também foram utilizados para avaliar a resposta fisiológica a imagens agradáveis, neutras e desagradáveis, calmas ou excitantes, em 66 sujeitos. Os resultados demonstraram variação conjunta entre as seguintes medidas fisiológico-comportamentais: (a) maior intensidade de ativação elétrica para o músculo zigomático (que ergue os cantos da boca e faz sorrir), menor ativação para o músculo corrugador do supercílio (que franze a testa e aproxima as sobrancelhas) e maior frequência cardíaca associadas com imagens agradáveis versus desagradáveis; (b) maiores índices de condutância elétrica da pele, com maiores níveis de excitação (arousal), independente de valência afetiva (Lang, Greenwald, Bradley, \& Hamm, 1993).

Frequência respiratória, eletromiografia da face, pressão sanguínea e temperatura corporal foram combinados com dados da atividade elétrica do cérebro (via EEG) e utilizados em um experimento que buscou relacionar respostas fisiológicas autonômicas a emoções despertadas por videoclipes musicais. Além da apresentação detalhada dos métodos de coleta e análise, importantes para a replicação do estudo, e de corroborar outros trabalhos que já haviam evidenciado a relação das respostas autonômicas com emoções, os autores desse estudo tornaram pública sua base de dados com 30 sujeitos que avaliaram 40 clipes, conclamando os pesquisadores na busca por métodos e algoritmos que expliquem a relação entre as valências dos videoclipes e as emoções resultantes (Koelstra, Soleymani, Yazdani, \& Nijholt, 2012). Em outro estudo, EEG, condutância da pele e da frequência cardíaca foram as medidas utilizadas para aferir a resposta neural decorrente do ato de assistir à televisão naturalmente, em 15 sujeitos, tendo-se evidenciado variações específicas nessas medidas, conforme o conteúdo a que assistiram e com o ato de se lembrar do mesmo ou não (Vecchiato et al., 2010).

Alterações do ritmo cardíaco, da condutância da pele e da frequência respiratória foram analisadas ainda em resposta a clipes de televisão com apelo afetivo negativo, como violência e tristeza, para examinar de que maneira a personalidade está ligada a respostas fisiológicas que sustentem as experiências emocionais vivenciadas, assim como a atenção despendida ao estímulo. Os resultados sugerem que filmes com apelo emocional tendem a gerar aumentos na excitação autonômica e na 
atenção. As respostas fisiológicas parecem ser influenciadas principalmente pelo próprio conteúdo, com a personalidade exercendo um papel secundário, especialmente no direcionamento da atenção (Brumbaugh, Kothuri, Marci, Siefert, \& Pfaff, 2013). De forma diferente, mas com o mesmo objetivo de investigar a relação entre personalidade e respostas autonômicas, 55 sujeitos foram expostos a imagens impressas com cargas afetivas positivas, negativas e neutras, e, nesse caso, a personalidade não foi capaz de influenciar suas respostas autonômicas causadas pelo estímulo (Mardaga, Laloyaux, \& Hansenne, 2006).

EEG, frequência cardíaca e condutância elétrica da pele, também foram as medidas utilizadas para investigar diferenças de resposta autonômica entre gêneros, ao expor consumidores aos mesmos comerciais publicitários de televisão (Vecchiato, Maglione, et al., 2014). Essas mesmas medidas também foram analisadas como indicadoras de confiança e preferência de voto em candidatos políticos (Vecchiato, Toppi, et al., 2014). Em outro estudo, a condutância elétrica da pele como medida de arousal foi utilizada em pesquisas de varejo, analisando como técnicas de organização do ponto de venda podem ser empregadas como ferramentas de estímulo para impactar o comportamento do consumidor (Groeppel-Klein, 2005).

A atividade facial muscular e a condutância elétrica da pele foram usadas simultaneamente ao EEG para investigar a resposta de mulheres a dois anúncios de televisão de um mesmo produto e com diferenças bastante sutis em apenas uma das cenas. Os resultados demonstraram reações autonômicas diferentes entre as duas peças publicitárias, mesmo que as participantes não percebessem conscientemente a diferença dos vídeos - conforme registrado em pós-testes (Ohme, Reykowska, Wiener, \& Choromanska, 2009).

A codificação da ação facial através do mapeamento das expressões faciais, principalmente ligadas às 6 reações emocionais básicas - raiva, nojo, medo, alegria, tristeza e surpresa -, permite outras análises e a identificação de diferentes padrões emocionais (Du, Tao, \& Martinez, 2014), que podem ser culturalmente específicos (Jack, Garrod, Yu, Caldara, \& Schyns, 2012). Diversos estudos evidenciam a relação de expressões faciais com comportamentos, em, com consumo. Isabela (2015) analisou, por meio de medidas eletromiográficas faciais, como consumidores percebem o constructo justiça de preço manipulando situações de terem pago mais ou pago menos que outros consumidores, considerando produtos hedônicos e utilitários e o tipo de apresentação de estímulos como figuras ou palavras.

Em um estudo que relacionou gostar/não gostar do sabor de bebidas com medidas de frequência cardíaca, condutância elétrica da pele, temperatura da pele e codificação facial, os resultados mostraram que gostar estava associado ao aumento na frequência cardíaca e da temperatura da pele, com emoções faciais mais neutras e negativamente relacionado a todas as demais expressões faciais, inclusive de felicidade, enquanto que intensidade de gostar estava associada com condutância elétrica da pele e com as expressões faciais de tristeza, raiva e surpresa (de Wijk, He, Mensink, Verhoeven, \& Graaf, 2014). As mesmas medidas foram investigadas com alimentos previamente definidos como saborosos ou não, mostrando comportamentos autonômicos distintos em cada situação de consumo (de Wijk, Kooijman, Verhoeven, Holthuysen, \& Graaf, 2012). Em outra pesquisa, a atividade elétrica dos músculos faciais foi utilizada, além da atividade elétrica neural e da condutância elétrica da pele, para investigar a influência de gênero e etnia no contexto do consumo de serviços (Boshoff, 2012).

A medida eletromiográfica, juntamente com o fluxo eletrodermal, foi utilizada para investigar a resposta autonômica em cenários de preços altos e baixos com marcas nacionais ou próprias. Os resultados sugerem que preços baixos e produtos de marcas nacionais induzem emoções mais positivas, com maior disparo elétrico do músculo zigomático (responsável por elevar/deprimir o ângulo da boca) em comparação a preços altos e produtos de marca própria (Somervuori \& Ravaja, 2013). A possibilidade de predição da efetividade de anúncios publicitários também já foi discutida utilizando a técnica da codificação facial (Lewinski, Fransen, \& Tan, 2014), assim como sua aplicação como ferramenta de mapeamento do comportamento do consumidor em pontos de venda (Bacivarov, Corcoran, \& Ionita, 2010). 
O reflexo do sobressalto, que é outra avaliação baseada no potencial elétrico muscular, foi testado no contexto de marketing quando se verificou a resposta de 21 indivíduos a marcas que gostavam ou não. Inicialmente os sujeitos foram orientados a classificar dentre 300 marcas internacionais quais gostavam e quais não gostavam. Após isso, a atividade muscular facial foi registrada enquanto avaliavam novamente algumas daquelas marcas, previamente selecionadas para serem marcas com carga afetiva positiva (gostavam) ou negativa (não gostavam). A intensidade do disparo elétrico muscular foi significativamente maior quando avaliavam marcas de que não gostavam, sugerindo que a medida pode ser um indicativo válido para o aspecto afetivo da atitude frente a marcas (Walla, Brenner, \& Koller, 2011).

A fim de relacionar dados neurobiológicos com o nível de stress de motoristas, Healey e Picard (2005) coletaram dados de eletrocardiograma, atividade elétrica muscular, frequência respiratória e condutância elétrica da pele em 24 sujeitos, por pelo menos 50 minutos enquanto dirigiam no entorno da cidade de Boston. Os resultados mostraram que, para a maioria dos motoristas estudados, condutividade da pele e frequência cardíaca foram as métricas mais estreitamente correlacionadas com o nível de stress do condutor, indicando que os sinais fisiológicos podem fornecer uma boa medida de stress. O stress causado pela tecnologia ou pela falha de determinado funcionamento esperado, chamado de tecnostress pelos pesquisadores de ciências da informação também foi estudado por meio de ferramentas neurobiológicas, como o hormônio cortisol salivar, que aumentou significativamente em usuários que vivenciaram situações de falha do sistema (Riedl, Kindermann, Auinger, \& Javor, 2012).

Níveis circulantes de hormônios caracterizam uma medida válida e bastante utilizada em pesquisas de comportamento, embora pouco aplicadas ao comportamento de consumo. Os níveis circulantes de cortisol aumentam em situação de stress agudo (Paris et al., 2010), e também estão relacionados a estados afetivos: níveis altos de cortisol salivar estão presentes em estados afetivos negativos, e os índices se reduzem a estados afetivos positivos (Smyth et al., 1997). A medida capilar do hormônio cortisol também pode ser um indicativo de stress e ansiedade crônicos (Steudte et al., 2011). Além do cortisol, a ocitocina - um hormônio produzido pelo hipotálamo e inicialmente relacionado à contração uterina - parece influenciar a empatia e a confiança, tornando sua atuação uma variável importante nas relações sociais e de consumo de maneira geral (Carvalho, 2016; Zak, Kurzban, \& Matzner, 2005), e ainda cortisol e testosterona podem ser marcadores para relacionamento interpessoal (Kornienko, Clemans, Out, \& Granger, 2014).

Estudos relacionando o comportamento de consumo feminino e a oscilação de hormônios importantes ao ciclo menstrual (estrogênio, progesterona e outros) têm sido publicados em periódicos científicos. Oscilações hormonais podem alterar a ingesta alimentar, embora a relação ainda seja controversa (Kuga, Ikeda, \& Suzuki, 1999; McVay, Copeland, Newman, \& Geiselman, 2012; Saad \& Stenstrom, 2012), e o mesmo acontece com decisões financeiras (Pine \& Fletcher, 2011) e comportamento social (Derntl, Hack, Kryspin-Exner, \& Habel, 2013; Jones et al., 2005; Markey \& Markey, 2011; Romans, Clarkson, Einstein, Petrovic, \& Stewart, 2012; Schwartz, Romans, Meiyappan, De Souza, \& Einstein, 2012).

Até mesmo estudos mais direcionados, como o marketing aplicado à disciplina de turismo, já tratam da influência de hormônios e neurotransmissores no comportamento de consumo, considerando essa uma boa ferramenta para a compreensão do comportamento dos seus consumidores (Koc \& Boz, 2014). Independente de objetivos e conclusões específicos, hormônios parecem ser marcadores biológicos confiáveis para pesquisas de comportamento.

Aplicações comportamentais para a medida de 2D:4D têm sido publicadas e são promissoras na busca da compreensão de motivações. Traços de personalidade parecem estar relacionados ao nível de exposição hormonal pré-natal, mais em mulheres que em homens (Fink et al., 2004), assim como a busca por sensações e a disposição a correr risco por elas, que é inversamente relacionada ao 2D:4D em homens - ou seja, quanto maior exposição à testosterona pré-natal, maior a disposição a correr riscos em busca de sensações (Fink et al., 2006). 
Disposição aos riscos social e recreacional, além de financeiro, também foram investigadas e parecem ter relação com esse indicador (Stenstrom et al., 2011). Em comerciantes, a exposição à testosterona pré-natal foi estatisticamente relacionada com medidas de desempenho do negócio, como lucro ou tempo de vida, além de relação entre disposição ao risco e à adaptabilidade ao ambiente (Coates et al., 2009).

Tomadas em conjunto as publicações descritas acrescentam teoria e método ao corpo teórico de marketing, enriquecem a área e aprofundam a investigação da relação entre os consumidores e os elementos de marketing. A abordagem multidisciplinar que se cria ao unir, além de outras disciplinas já integradas, também a neurociência nas investigações de marketing, permite que novas facetas dessa relação sejam conhecidas.

\section{Agenda de pesquisa}

Considerando diversas características das técnicas neurofisiológicas segundo sua finalidade, forma de mensuração e procedimentos de coleta de dados, as diferentes ferramentas apresentadas neste estudo atendem distintos objetivos. Essa consideração sugere que um fator relevante a ser considerado é a possibilidade de uso sincronizado de múltiplas ferramentas, aspecto este que possibilita análises complementares, de modo a ter-se uma visão mais integrada do fenômeno em estudo. Outro ponto comum no uso dessas ferramentas é a composição de times multidisciplinares de pesquisa e da condução ética da mesma (Isabela, Mazzon, \& Dimoka, 2015).

Os métodos que registram atividade metabólica do cérebro (PET e fMRI) e que registram a atividade elétrica do mesmo (EEG, EMT, TEE e MEG), embora registrem maiores dificuldades de coleta de dados enquanto tempo, custo e perfil da equipe de análise, são promissores em termos de tempo de resultado e assertividade de respostas cerebrais (Isabela, 2015). Podem ser úteis em estudos que aprofundem relações de processamento de informação cerebral como a reação autonômica frente a imagens, sons, filmes, material publicitário, dentre outros, que objetivem explorar variáveis dependentes, como interesse, preferência e tipos de memória (Vecchiato et al., 2010). Variações de gênero nesse contexto podem ser interessantes em estudos futuros (Vecchiato, Maglione, et al., 2014). A exploração sobre elementos que exploram diferentes tipos de memória é um caminho a ser percorrido, já que os métodos mencionados permitem o registro sobre as áreas cerebrais ativadas, bem como a correlação dessas com o comportamento posterior. Estudos longitudinais podem demonstrar a evolução do aprendizado e o perfil de memória sobre diferentes estímulos.

Ferramentas que registram respostas secundárias a comandos neuronais (eye-tracker, condutância elétrica da pele, EMG, controle hormonal, dentre outros), possuem menor tempo e custo de coleta, e podem ser aplicadas de forma simultânea e sincronizada, fato que amplia a capacidade de explicação dos achados (Koelstra et al., 2012). A exploração de emoções básicas via EMG, somadas à condutância elétrica da pele, podem ser úteis na medida de respostas autonômicas na avaliação de diferentes estímulos comerciais, como anúncios audiovisuais, sons, imagens, manipulação de elementos visuais, como cores, palavras, dentre outros. Podem ainda estar associados à indicação de preferências e elementos mais estimulantes.

A pré-disposição de avaliação de marcas e estímulos publicitários, como preço, argumentos de venda, narrativas, podem ser explorados para melhor compreensão das reações comportamentais. Somervuori e Rvaja (2013) demonstraram correlações significativas entre preços baixos e marcas conhecidas com emoções básicas positivas. Questões de gênero e etnias também podem ser exploradas. Avaliações acerca do perfil e da composição das escolhas do consumidor podem ser exploradas. Além das avaliações de autodeclaração sobre as intenções e as atitudes de consumo, a criatividade em métodos mistos pode ser um caminho a ser explorado com provocações de escolhas concretas para o consumidor, aproximando as situações de análise à realidade de mercado. Diferentes correntes teóricas podem ser exploradas acerca do processamento de informações e tomada de decisão, como a teoria dos jogos, a teoria de sinalização, dentre outras. 
Estudos futuros podem também aprofundar o conhecimento sobre a maneira pela qual modificações hormonais como as que acontecem no stress podem alterar respostas comportamentais enquanto interesse de consumo, níveis de atenção e alteração de emoções básicas. Estado de stress agudo diminui a capacidade cognitiva do consumidor, fato que pode dificultar a avaliação entre marcas e características de produtos. Esse tipo de situação pode acontecer em diferentes tipos de varejo onde, sob pressão, como música, excesso de pessoas no ambiente, tempo escasso para compra, dentre outros, o consumidor pode ser levado a ter reações de escolha e consumo orientado por atalhos mentais. Compreender melhor os meandros dessas relações pode contribuir com teorias acerca do comportamento de consumo e da tomada de decisão. Outras questões hormonais interessantes sobre o comportamento feminino estão associadas às variações do ciclo hormonal (Pastore, FranciscoMaffezzolli, Silva, \& Baptista, 2016).

A influência hormonal também é percebida não só nos níveis circulantes, mas também no impacto de níveis pré-natais, como no caso do Digit Ratio. Investigar o impacto comportamental dessa influência pode ajudar a compreender melhor as motivações de consumo, em especial no consumo de produtos de conquista e, na prática, pode ajudar gestores a posicionarem produtos e encontrar argumentos de persuasão mais eficientes.

Outros temas comuns na gestão de marketing, como teste sobre design de embalagem, lançamento de produtos, estudos de ambiente de varejo, experiências de compra online, relacionamento com marcas, estratégias de comunicação, decisões financeiras de investimento, podem ser explorados com uso de ferramentas neurobiológicas, combinadas entre si e com técnicas tradicionais de pesquisa (survey, observação, grupos focais, etnografia, dentre outras).

Sobre questões metodológicas, em geral, o uso de biomarcadores está associado a experimentos laboratoriais, já que o nível de controle de variáveis externas é considerável e fundamental para a validade e a confiabilidade das mensurações efetuadas. $\mathrm{O}$ ambiente, ruídos, interferências externas, dentre outros, são elementos que podem criar vieses nos dados, dificultando a coleta, a análise dos dados e a interpretação dos resultados.

\section{Considerações sobre Ética na Pesquisa com Biomarcadores}

A utilização de indicadores biológicos exige dedicação do pesquisador para que se cumpram todas as diretrizes éticas cabíveis. Pesquisas dessa natureza são típicas de outras áreas do conhecimento e o desafio dos pesquisadores, sob essa perspectiva, é menor que o alvoroço incentivado por críticos à aproximação do marketing e da neurociência, uma vez que já existem protocolos éticos e condutas a serem seguidas. As preocupações éticas sobre a participação de sujeitos condicionada à total liberdade e esclarecimento metodológico prévio, a privacidade dos dados coletados ou sua possível utilização para predizer comportamento estão presentes nos diversos tipos de condução de pesquisa, desde as consideradas tradicionais, via autorrelato, até o uso de biomarcadores (Lee et al., 2010). A expressão buy button já foi citada diversas vezes como referência ao uso de dados nurobiológicos em pesquisas de marketing e o poder persuasivo que em tese deriva dessas técnicas (Murphy, Illes, \& Reiner, 2008), mas se faz inadequada se conduzidos com critério os procedimentos e cuidados éticos inerentes à pesquisa.

Quando se trata de regulamentação e controle, cabe aos órgãos institucionais - Comitês de Ética em Pesquisa - garantirem o cumprimento integral das diretrizes que, assim como as técnicas e teorias utilizadas, devem buscar inspiração nos protocolos adotados pelas ciências biológicas e da saúde. Um dos principais documentos norteadores dessa natureza é o Belmont Report (Ryan, 1979), que especifica princípios para a proteção de sujeitos humanos em pesquisas, incluindo grande diversidade de tipos de dados. O documento ressalta, dentre outros, ser fundamental a avaliação prévia de todos os protocolos de pesquisa por outros pesquisadores não envolvidos com o projeto, que possam apreciá-lo de maneira imparcial. A adoção desse protocolo pelos Comitês de Universidades também para pesquisas na área das ciências sociais aplicadas parece bastante promissora. 
No que diz respeito aos cuidados que empresas de pesquisa devem tomar ao utilizar biomarcadores, Stanton, Sinnott-Armstrong e Huettel (2017) discutem a necessidade de rigor metodológico, revisão por pares na academia e na indústria e transparência dos protocolos utilizados até dados e resultados - para a confiabilidade das pesquisas e a segurança dos sujeitos participantes. Iniciativas como o Neuro Standards Collaboration Project, da Advertising Research Foundation (ARF, www.thearf.org) - uma revisão independente dos métodos de pesquisa adotados com diretrizes de condutas - são passos iniciais na busca por mais rigor ético no mercado e podem servir de exemplos a órgãos regulamentadores públicos. Além disso, acredita-se que a parceria entre empresas e universidades para a condução conjunta de pesquisas seja um elemento importante para garantir a condução ética de pesquisas dessa natureza.

No Brasil e no mundo, existem iniciativas governamentais para garantir o cuidado ético e a proteção de sujeitos participantes de pesquisas, sejam acadêmicas ou de mercado. A Comissão Nacional de Ética em Pesquisa (CONEP), por exemplo, vinculada ao Ministério da Saúde brasileiro, hospeda o Sistema Nacional de Informação sobre Ética em Pesquisa Envolvendo Seres Humanos (SISNEP http://www.saude.go.br/sisnep,www.niehs.nih.gov), que é uma entidade importante na disseminação de conhecimento e na regulamentação de pesquisas envolvendo animais e seres humanos. O Departamento de Saúde e Serviços Humanos do governo dos EUA possui atividade semelhante, através do National Institute of Environmental Health Sciences (NIEHS - www.niehs.nih.gov), que atua como orientador e regulamentador de atividades de pesquisa. Alguns dos princípios éticos que devem ser observados segundo essas duas entidades (NIEHS e SISNEP) são: honestidade, objetividade, integridade, abertura de dados e protocolos, confidencialidade e publicação responsável.

Por fim, ressalta-se que o grande desafio ético na condução de pesquisas com biomarcadores está na coleta e no armazenamento dos dados obtidos. Há o receio de que, por se tratar do mapeamento de respostas fisiológicas, pesquisadores poderiam coletar dados sem o consentimento dos sujeitos participantes, invadindo, assim, o princípio da privacidade. Essa possibilidade é sanada ao exigir o cumprimento de protocolos rígidos de pesquisa, tanto na academia quanto no mercado.

\section{Considerações Finais}

Embora o comportamento humano seja complexo demais para ser resumido à leitura da atividade cerebral ou de reações autonômicas diversas, se aplicadas corretamente, as ferramentas advindas da neurociência podem auxiliar na compreensão do pensar e do agir dos consumidores. Afinal, "ter novas percepções do conhecimento não é algo novo, mas encontrar novas interconexões entre estas percepções é benéfico para a criação e disseminação do conhecimento" (Butler, 2008, p. 1).

A neurociência pode prover conhecimentos e ferramentas enriquecedoras, mas ainda se apresenta como uma lente um pouco desfocada e obtusa, através da qual não se sabe bem o que está enxergando: falta compreensão biológica, o que pode levar a conclusões equivocadas. O caminho para superar tal dificuldade passa pela formação de equipes multidisciplinares, parcerias público-privadas para financiamento de pesquisas e por disposição para pensar diferente da forma tradicional, como têm sido feitas as pesquisas de marketing.

É fundamental ao avanço das teorias de marketing que se abram novas possibilidades de multidisciplinariedade, fomentando diferentes visões aos comportamentos observados. Quando se trata de compreender o comportamento de seres humanos, mercadólogos e administradores se beneficiam dos conhecimentos de psicólogos, sociólogos, antropólogos, neurocientistas e todos os demais pesquisadores de diversas outras áreas que, em alguma instância, observam comportamentos. É preciso ampliar os horizontes conhecendo as possibilidades e os limites de cada área para que o corpo teórico cresça e se solidifique. Foi sob essa premissa que este trabalho foi escrito. 
Outro tema em permanente discussão para pesquisas com seres humanos trata dos limites éticos e morais da condução de processos e protocolos, análises, interpretação, armazenamento e finalidade de uso dos dados. No cenário brasileiro, o SISNEP tem o objetivo de assegurar, via CONEP (Comissão Nacional de Ética em Pesquisa) e CEP (Comitê de Ética em Pesquisa), o cuidado devido na condução de pesquisas. Academia e indústria estão regidos pelas mesmas regras de condução de pesquisa. Entretanto, outra questão moral em discussão versa sobre a capacidade preditiva de certos dados, coletados de forma consensual, mas não necessariamente consciente, capaz de induzir comportamentos intencionais em benefício particular de uma organização. Aqui talvez estejam sediadas reflexões sobre os limites éticos de uso de dados com essa natureza. Semelhante discussão se faz no âmbito do uso de informações digitais, chamadas de dados abertos, porém, sem conclusões definitivas. Estas questões não são recentes na pesquisa, mas se tornam relevantes e em foco devido à amplitude e à crescente tecnologia empregada nos dados.

Ao se colocarem diferentes ferramentas neurocientíficas em perspectiva, percebe-se que algumas questões sobre tempo, custo, tratamento dos dados, formação de equipes e objetivos de pesquisa são norteadores sobre os caminhos mais apropriados de investigação. O ponto comum de preocupação reside acerca de competências e ética na condução dos procedimentos, calibragem dos equipamentos utilizados e sincronização de medidas quando os estudos forem complementares e utilizarem diferentes mensurações de forma simultânea. Por fim, recorda-se que a relevância do suporte teórico em pesquisas futuras deve se sobressair em relação às ferramentas. Primeiro o sentido, o conceito e as relações, a sustentação de hipóteses e, só depois, a métrica.

\section{Referências}

AbcMed - Informações sobre sua Saúde. (2013, setembro 20). Eletroencefalograma: como é feito? Como se preparar para o exame? Quais são as complicações? Recuperado em 7 julho, 2016, de http://www.abc.med.br/p/exames-e-

procedimentos/347519/eletroencefalograma+como+e+feito+como+se+preparar+para+o+exame +quais+sao+as+complicacoes.htm

AbcMed - Informações sobre sua Saúde. (2013, setembro 30). Nevralgia ou neuralgia: o que é isso? Recuperado em 7 julho, 2016, de http://www.abc.med.br/p/sinais.-sintomas-edoencas/369059/nevralgia+ou+neuralgia+o+que+e+isso.htm

AbcMed - Informações sobre sua Saúde. (2015, janeiro 21). PET Scan: o que é? Por que fazer? Como é feita? Quem deve e quem não deve fazer? Quais são as possíveis complicações? Recuperado em 7 julho, 2016, de http://www.abc.med.br/p/exames-eprocedimentos $/ 740267 /$ pet+scan+o+que+e+por+que+fazer+como+e+feita+quem+deve+e+que $\mathrm{m}+$ nao+deve+fazer+quais+sao+as+possiveis+complicacoes.htm

Austin, E. J., Manning, J. T., McInroy, K., \& Mathews, E. (2002). A preliminary investigation of the associations between personality, cognitive ability and digit ratio. Personality and Individual Differences Personality and Individual Differences, 33(7), 1115-1124. http://dx.doi.org/10.1016/S0191-8869(02)00002-8

Bacivarov, I., Corcoran, P., \& Ionita, M. (2010). Smart cameras: 2D affine models for determining subject facial expressions. IEEE Transactions on Consumer Eletronics, 56(2), 289-297. http://dx.doi.org/10.1109/TCE.2010.5505930

Boshoff, C. (2012). A neurophysiological assessment of consumers' emotional responses to service recovery behaviors: The impact of ethnic group and gender similarity. Journal of Service Research, 15(4), 401-413. http://dx.doi.org/10.1177/1094670512453879 
Boxtel, A. van (2010, August). Facial EMG as a tool for inferring affective states. Proceedings of Measuring Behavior, Eindhoven, NLD, 7.

Braithwaite, J. J., Watson, D. G., Jones, R., \& Rowe, M. (2013). A guide for analysing electrodermal Activity (EDA) \& skin conductance responses (SCRs) for Psychological Experiments. Retrieved from https://www.biopac.com/wp-content/uploads/EDA-SCR-Analysis.pdf

Brumbaugh, C. C., Kothuri, R., Marci, C., Siefert, C., \& Pfaff, D. D. (2013). Physiological correlates of the big 5: Autonomic responses to video presentations. Applied Psychophysiology and Biofeedback, 38(4), 293-301. http://dx.doi.org/10.1007/s10484-013-9234-5

Butler, M. J. R. (2008). Neuromarketing and the perception of knowledge. Journal of Consumer Behaviour, 7(4/5), 415-419. http://dx.doi.org/10.1002/cb.260

Centro Médico Sorocaba. (n.d.). Exames. Recuperado em 7 julho, 2016, de https://www.centromedicosorocaba.com.br/exames

Coates, J. M., Gurnell, M., \& Rustichini, A. (2009). Second-to-fourth digit ratio predicts success among high-frequency financial traders. Proceedings of the National Academy of Sciences of the United States of America, 106(2), 623-628. http://dx.doi.org/10.1073/pnas.0810907106

Criswell, E. (2011). Cram's introduction to surface electromyografy (2nd ed.). Sudbury,MA: Jones and Bartlett Publishers.

Dart, A., Du, X., \& Kingwell, B. (2002). Gender, sex hormones and autonomic nervous control of the cardiovascular system. Cardiovascular Research, 53(3), 678-687.

Derntl, B., Hack, R. L., Kryspin-Exner, I., \& Habel, U. (2013). Association of menstrual cycle phase with the core components of empathy. Hormones and Behavior, 63(1), 97-104. http://dx.doi.org/10.1016/j.yhbeh.2012.10.009

Du, S., Tao, Y., \& Martinez, A. M. (2014). Compound facial expressions of emotion. Proceedings of the National Academy of Sciences of the United States of America, 111(15), E1454-62. https://doi.org/10.1073/pnas.1322355111

Ekman, P. (2006). Darwin, deception, and facial expression. Annals of the New York Academy of Sciences, 1000(1), 205-221. http://dx.doi.org/10.1196/annals.1280.010

Ekman, P., \& Friesen, W. V. (1982). Felt, false, and miserable smiles. Journal of Nonverbal Behavior, 6(4), 238-252. https://doi.org/10.1007/BF00987191

Ekman, P., \& Rosenberg, E. L. (1997). What the face reveals: Basic and applied studies of spontaneous expression using the facial action coding system (FACS). New York: Oxford University Press.

Fink, B., Manning, J. T., \& Neave, N. (2004). Second to fourth digit ratio and the "big five" personality factors. Personality and Individual Differences, 37(3), 495-503. http://dx.doi.org/10.1016/j.paid.2003.09.018

Fink, B., Neave, N., Laughton, K., \& Manning, J. T. (2006). Second to fourth digit ratio and sensation seeking. Personality and Individual Differences, 41(7), 1253-1262. http://dx.doi.org/10.1016/j.paid.2006.05.002

Gakhal, B., \& Senior, C. (2008). Exarnining the influence of fame in the presence of beauty: An electroderrnal "neurornarketing" study. Journal of Consumer Research, 7(4/5), 331-341. http://dx.doi.org/10.1002/cb.255

Groeppel-Klein, A. (2005). Arousal and consumer in-store behavior. Brain Research Bulletin, 67(5), 428-437. http://dx.doi.org/10.1016/j.brainresbull.2005.06.012 
Habel, U., Windischberger, C., Derntl, B., Robinson, S., Kryspin-Exner, I., Gur, R. C., \& Moser, E. (2007). Amygdala activation and facial expressions: Explicit emotion discrimination versus implicit emotion processing. Neuropsychologia, 45(10), 2369-2377. http://dx.doi.org/10.1016/j.neuropsychologia.2007.01.023

Healey, J. A., \& Picard, R. W. (2005). Detecting stress during real-world driving tasks using physiological sensors. IEEE Transactions on Intelligent Transportation Systems, 6(2), 156-166. http://dx.doi.org/10.1109/TITS.2005.848368

Hill, D. (2003). Tell me no lies: Using science to connect with consumers. Journal of Interactive Marketing, 17(4), 61-72. http://dx.doi.org/10.1002/dir.10068

Hubert, M. (2010). Does neuro economics give new impetus to economic and consumer research? Journal of Economic Psychology, 31(5), 812-817. http://dx.doi.org/10.1016/j.joep.2010.03.009

Isabela, G. (2015). Hedonic and utilitarian purchases and construal level theory in the perception of justice and price fairness: Behavioral and physiological perspectives (Tese de doutorado). Universidade de São Paulo, São Paulo, SP, Brasil.

Isabela, G., Mazzon, J. A., \& Dimoka, A. (2015). Culture differences, difficulties, and challenges of the neurophysiological methods in marketing research. Journal of Internacional Consumer Marketing, 27(5), 1-18. http://dx.doi.org/10.1080/08961530.2015.1038761

Jack, R. E., Garrod, O. G. B., Yu, H., Caldara, R., \& Schyns, P. G. (2012). Facial expressions of emotion are not culturally universal. Proceedings of the National Academy of Sciences of the United States of America, 109(19), 7241-7244. http://dx.doi.org/10.1073/pnas.1200155109

Javor, A., Koller, M., Lee, N., Chamberlain, L., \& Ransmayr, G. (2013). Neuromarketing and consumer neuroscience: Contributions to neurology. BMC Neurology, 13(1), 1-12. http://dx.doi.org/10.1186/1471-2377-13-13

Jones, B. C., Little, A. C., Boothroyd, L., DeBruine, L. M., Feinberg, D. R., Smith, M. J. L., Cornwellc, R. E., Moorec, F. R., \& Perrett, D. I. (2005). Commitment to relationships and preferences for femininity and apparent health in faces are strongest on days of the menstrual cycle when progesterone level is high. Hormones and Behavior, 48(3), 283-290. http://dx.doi.org/10.1016/j.yhbeh.2005.03.010

Kenning, P. H., \& Plassmann, H. (2008). How neuroscience can inform consumer research. IEEE Transactions on Neural Systems and Rehabilitation Engineering, 16(6), 532-538. http://dx.doi.org/10.1109/TNSRE.2008.2009788

Kenning, P., \& Plassmann, H. (2005). NeuroEconomics: An overview from an economic perspective. Brain Research Bulletin, 67(5), 343-354. http://dx.doi.org/10.1016/j.brainresbull.2005.07.006

Koc, E., \& Boz, H. (2014). Psychoneurobiochemistry of tourism marketing. Tourism Management, 44, 140-148. http://dx.doi.org/10.1016/j.tourman.2014.03.002

Koelstra, S., Soleymani, M., Yazdani, A., \& Nijholt, A. (2012). DEAP: A database for emotion analysis using physiological signals. IEEE Transactions on Affective Computing, 3(1), 18-31. http://dx.doi.org/10.1109/T-AFFC.2011.15

Koller, M., \& Walla, P. (2012). Measuring affective information processing in information systems and consumer research - introducing startle reflex modulation. Proceedings of the International Conference of Information Systems, Orlando, Florida, USA, 33.

Kornienko, O., Clemans, K. H., Out, D., \& Granger, D. A. (2014). Hormones, behavior, and social network analysis: Exploring associations between cortisol, testosterone, and network structure. Hormones and Behavior, 66(3), 534-544. http://dx.doi.org/10.1016/j.yhbeh.2014.07.009 
Kreibig, S. D. (2010). Autonomic nervous system activity in emotion: A review. Biological Psychology, 84(3), 394-421. http://dx.doi.org/10.1016/j.biopsycho.2010.03.010

Kuga, M., Ikeda, M., \& Suzuki, K. (1999). Gustatory changes associated with the menstrual cycle. Physiology \& Behavior, 66(2), 317-322. http://dx.doi.org/10.1016/S0031-9384(98)00307-2

Lang, P. J., Greenwald, M. K., Bradley, M. M., \& Hamm, A. O. (1993). Looking at pictures: Affective, facial, visceral, and behavioral reactions. Psychophysiology, 30(3), 261-273. http://dx.doi.org/10.1111/j.1469-8986.1993.tb03352.x

Lee, N., Butler, M., \& Senior, C. (2010). The brain in business: The case for organisational cognitive neuroscience? Nature Precedings. 49(3), 129-131. http://dx.doi.org/10.1007/s12642-010-0033-8

Lent, R. (2004). Cem bilhões de neurônios? Conceitos fundamentais de neurociência (2a ed.). Rio de Janeiro: Atheneu.

Lewinski, P., Fransen, M. L., \& Tan, E. S. H. (2014). Predicting advertising effectiveness by facial expressions in response to amusing persuasive stimuli. Journal of Neuroscience, Psychology, and Economics, 7(1), 1-14. http://dx.doi.org/10.1037/npe0000012

Lutchmaya, S., Baron-Cohen, S., Raggatt, P., Knickmeyer, R., \& Manning, J. T. (2004). 2nd to 4th digit ratios, fetal testosterone and estradiol. Early Human Development, 77(1/2), 23-28. http://dx.doi.org/10.1016/j.earlhumdev.2003.12.002

Mardaga, S., Laloyaux, O., \& Hansenne, M. (2006). Personality traits modulate skin conductance response to emotional pictures: An investigation with Cloninger's model of personality. Personality and Individual Differences, 40(8), 1603-1614. https://doi.org/10.1016/j.paid.2005.12.006

Markey, P., \& Markey, C. (2011). Changes in women's interpersonal styles across the menstrual cycle. Journal of Research in Personality, 45(5), 493-499. http://dx.doi.org/10.1016/j.jrp.2011.06.005

McVay, M. A., Copeland, A. L., Newman, H. S., \& Geiselman, P. J. (2012). Food cravings and food cue responding across the menstrual cycle in a non-eating disordered sample. Appetite, 59(2), 591-600. http://dx.doi.org/10.1016/j.appet.2012.07.011

Mesquita, B. (2016). The legacy of Nico H. Fridja (1927 - 2015). Cognition and Emotion, 30(4) 603608. http://dx.doi.org/10.1080/02699931.2015.1132681

Murphy, E. R., Illes, J., \& Reiner, P. B. (2008). Neuroethics of neuromarketing. Journal of Consumer Behaviour, 7(4/5), 293-302. http://dx.doi.org/10.1002/cb.252

Nepomuceno, M. V., Saad, G., Stenstrom, E., Mendenhall, Z., \& Iglesias, F. (2016). Testosterone at your fingertips: Digit ratios (2D:4D and rel2) as predictors of courtship-related consumption intended to acquire and retain mates. Journal of Consumer Psychology, 26(2), 231-244. http://dx.doi.org/10.1016/j.jcps.2015.05.007

Ohme, R., Reykowska, D., Wiener, D., \& Choromanska, A. (2009). Analysis of neurophysiological reactions to advertising stimuli by means of EEG and galvanic skin response measures. Journal of Neuroscience, Psychology, and Economics, 2(1), 21-31. http://dx.doi.org/10.1037/a0015462

Paris, J. J., Franco, C., Sodano, R., Freidenberg, B., Gordis, E., Anderson, D. A., Forsyth, J. P., Wulfert, E., \& Frye, C. A. (2010). Sex differences in salivary cortisol in response to acute stressors among healthy participants, in recreational or pathological gamblers, and in those with posttraumatic stress disorder. Hormones and Behavior, 57(1), 35-45. http://dx.doi.org/10.1016/j.yhbeh.2009.06.003 
Pastore, C. M. de A., Francisco-Maffezzolli, E. C., Silva, W. V. da, \& Baptista, P. de P. (2016). Premenstrual period: Do women really consume more? Journal of Consumer Behaviour, 16(1), 4250. http://dx.doi.org/10.1002/cb.1601

Pastore, C. M. de A., Nepomuceno, M. V., Stenstrom, E., \& Francisco-Maffezzolli, E. C. (2016). The association between digit ratios and conspicuous consumption, and the moderating role of intrasexual competition. ACR Conference (Vol. 53). http://dx.doi.org/10.1017/CBO9781107415324.004

Perloff, D., Grim, C., Flack, J., \& Frohlich, E. (1993). Human blood pressure determination by sphygmomanometry. Circulation, 88(5), 2460-2471. http://dx.doi.org/10.1161/01.CIR.88.5.2460

Pine, K. J., \& Fletcher, B. (C). (2011). Women's spending behaviour is menstrual-cycle sensitive. $\begin{array}{llll}\text { Personality and Individual } & \text { Differences, } & 50(1), & \text { 74-78. }\end{array}$ http://dx.doi.org/10.1016/j.paid.2010.08.026

Plassmann, H., Ramsøy, T. Z., \& Milosavljevic, M. (2012). Branding the brain: A critical review and

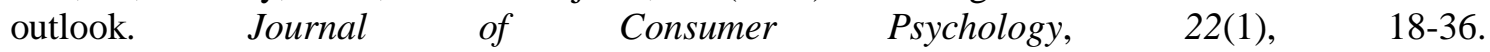
http://dx.doi.org/10.1016/j.jcps.2011.11.010

Riedl, R., Kindermann, H., Auinger, A., \& Javor, A. (2012). Technostress from a Neurobiological Perspective. Business \& Information Systems Engineering, 4(2), 61-69. http://dx.doi.org/10.1007/s12599-012-0207-7

Romans, S., Clarkson, R., Einstein, G., Petrovic, M., \& Stewart, D. (2012). Mood and the menstrual cycle: A review of prospective data studies. Gender Medicine, 9(5), 361-384. http://dx.doi.org/10.1016/j.genm.2012.07.003

Ryan, K. J., Brady, J. V., Cooke, R. E., Height, D. I., Jonsen, A. R., King, P., Lebacqz, K., Louisell, D. W., Seldin, D. W., Stellar, E., \& Turtle, R. H. (1979, April 18). The Belmont report: Ethical principles and guidelines for the protection of human subjects of research. Retrieved from https://www.hhs.gov/ohrp/sites/default/files/the-belmont-report-508c_FINAL.pdf

Saad, G., \& Stenstrom, E. (2012). Calories, beauty, and ovulation: The effects of the menstrual cycle on food and appearance-related consumption. Journal of Consumer Psychology, 22(1), 102-113. http://dx.doi.org/10.1016/j.jcps.2011.10.001

Schwartz, D. H., Romans, S. E., Meiyappan, S., De Souza, M. J., \& Einstein, G. (2012). The role of ovarian steroid hormones in mood. Hormones and Behavior, 62(4), 448-454. http://dx.doi.org/10.1016/j.yhbeh.2012.08.001

Shiv, B., Bechara, A., Levin, I., Alba, J. W., Bettman, J. R., Dube, L., Isen, A., Mellers, B., Smidts, A., Grant, S. J., \& Mcgraw, A. P. (2005). Decision Neuroscience. Marketing Letters, 16(3/4), 375386. http://dx.doi.org/10.1007/s11002-005-5899-8

Smyth, J. M., Ockenfels, M. C., Gorin, A. A., Catley, D., Porter, L. S., Kirschbaum, C., Hellhammer, D. H., \& Stone, A. A. (1997). Individual differences in the diurnal cycle of cortisol. Psychoneuroendocrinology, 22(2), 89-105. http://dx.doi.org/10.1016/S0306-4530(96)00039-X

Somervuori, O., \& Ravaja, N. (2013). Purchase behavior and psychophysiological responses to different price levels. Psychology \& Marketing, 30(6), 479-489. http://dx.doi.org/10.1002/mar

Stanton, S. J., Sinnott-Armstrong, W., \& Huettel, S. A. (2017). Neuromarketing: Ethical implications of its use and potential misuse. Journal of Business Ethics, 144(4), 799-811. http://dx.doi.org/10.1007/s10551-016-3059-0

Stenstrom, E., Saad, G., Nepomuceno, M. V., \& Mendenhall, Z. (2011). Testosterone and domainspecific risk: Digit ratios (2D:4D and rel2) as predictors of recreational, financial, and social risk- 
taking behaviors. Personality and Individual Differences, 51(4), 412-416. http://dx.doi.org/10.1016/j.paid.2010.07.003

Steudte, S., Stalder, T., Dettenborn, L., Klumbies, E., Foley, P., Beesdo-Baum, K., \& Kirschbaum, C. (2011). Decreased hair cortisol concentrations in generalised anxiety disorder. Psychiatry Research, 186(2/3), 310-314. http://dx.doi.org/10.1016/j.psychres.2010.09.002

Vecchiato, G., Astolfi, L., De Vico Fallani, F., Cincotti, F., Mattia, D., Salinari, S., Soranzo, R., \& Babiloni, F. (2010). Changes in brain activity during the observation of TV commercials by using EEG, GSR and HR measurements. Brain Topography, 23(2), 165-179. http://dx.doi.org/10.1007/s10548-009-0127-0

Vecchiato, G., Maglione, A. G., Cherubino, P., Wasikowska, B., Wawrzyniak, A., Latuszynska, A., Latuszynska, M., Nermend, K., Graziani, I., Leucci, M. R., Trettel, A., \& Babiloni, F. (2014). Neurophysiological tools to investigate consumer's gender differences during the observation of TV commercials. Computational and Mathematical Methods in Medicine, 2014, 1-12. http://dx.doi.org/10.1155/2014/912981

Vecchiato, G., Toppi, J., Maglione, A. G., Olejarczyk, E., Astolfi, L., Mattia, D., Colosimo. A., \& Babiloni, F. (2014). Neuroelectrical correlates of trustworthiness and dominance judgments related to the observation of political candidates. Computational and Mathematical Methods in Medicine, 2014, 1-20. http://dx.doi.org/10.1155/2014/434296

Walla, P., Brenner, G., \& Koller, M. (2011). Objective measures of emotion related to brand attitude: A new way to quantify emotion-related aspects relevant to marketing. PloS One, 6(11), 1-7. http://dx.doi.org/10.1371/journal.pone.0026782

Weierich, M. R., Wright, C. I., Negreira, A., Dickerson, B. C., \& Barrett, L. F. (2010). Novelty as a dimension in the affective brain. NeuroImage, 49(3), 2871-2878. http://dx.doi.org/10.1016/j.neuroimage.2009.09.047

Wijk, R. A. de, Kooijman, V., Verhoeven, R. H. G., Holthuysen, N. T. E., \& Graaf, C. de (2012). Autonomic nervous system responses on and facial expressions to the sight, smell, and taste of liked and disliked foods. Food Quality and Preference, 26(2), 196-203. http://dx.doi.org/10.1016/j.foodqual.2012.04.015

Wijk, R. A. de, He, W., Mensink, M. G. J., Verhoeven, R. H. G., \& Graaf, C. de (2014). ANS responses and facial expressions differentiate between the taste of commercial breakfast drinks. PloS One, 9(4), e93823. http://dx.doi.org/10.1371/journal.pone.0093823

Yan, X., Andrews, T. J., Jenkins, R. \& Young, A. W. (2016). Cross-cultural differences and similarities underlying other-race effects for facial identity and expression, The Quarterly Journal of Experimental Psychology, 69(7), 1247-1254. http://dx.doi.org/10.1080/17470218.2016.1146312

Yoon, C., Gonzalez, R., Bechara, A., Berns, G. S., Dagher, A. A., Dubé, L., Scott, A., Huettel, S. A., Kable, J. W., Liberzon, I., Plassmann, H., Smidts, A., \& Spence, C. (2012). Decision neuroscience and consumer decision making. Marketing Letters, 23(2), 473-485. http://dx.doi.org/10.1007/s11002-012-9188-z

Zak, P. J., Kurzban, R., \& Matzner, W. T. (2005). Oxytocin is associated with human trustworthiness. Hormones and Behavior, 48(5), 522-527. http://dx.doi.org/10.1016/j.yhbeh.2005.07.009

Zurawicki, L. (2010). Neuromarketing, exploring the brain of the consumer. Berlin: Springer-Verlag. 


\section{Dados dos Autores}

Cristina Maria de Aguiar Pastore

Rua Imaculada conceição, 1115, 80215-901, Curitiba, PR, Brasil: E-mail: crispastore@ gmail.com. https://orcid.org/00000003-4920-0997

Eliane Cristine Francisco Maffezzolli

Rua Imaculada conceição, 1115, 80215-901, Curitiba, PR, Brasil: E-mail: eliane.francisco@ pucpr.br. http://orcid.org/00000002-4775-0824

José Afonso Mazzon

Av. Prof. Luciano Gualberto, 908, Sala E-187, 05508-900, São Paulo, SP, Brasil: E-mail: jamazzon@usp.br. http://orcid.org/0000-0003-1556-520X 